CARNETS OE Carnets de géographes

GÉOGRAPHES

$1 \mid 2010$

Varia

\title{
Perspectives géographiques pour la musique
}

La mobilisation du flamenco comme ressource territoriale

\section{Nicolas Canova}

\section{(2) OpenEdition}

Journals

Édition électronique

URL : http://journals.openedition.org/cdg/1819

DOI : $10.4000 / \mathrm{cdg} .1819$

ISSN : 2107-7266

Éditeur

UMR 245 - CESSMA

Référence électronique

Nicolas Canova, «Perspectives géographiques pour la musique », Carnets de géographes [En ligne],

1 | 2010, mis en ligne le 01 octobre 2010, consulté le 07 mai 2019. URL : http://

journals.openedition.org/cdg/1819; DOI : 10.4000/cdg.1819

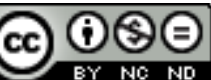

La revue Carnets de géographes est mise à disposition selon les termes de la Licence Creative Commons Attribution - Pas d'Utilisation Commerciale - Pas de Modification 4.0 International. 


\title{
PERSPECTIVES GEOGRAPHIQUES POUR LA MUSIQUE La mobilisation du flamenco comme ressource territoriale
}

\author{
Nicolas Canova \\ canovanicolas@yahoo.fr \\ Université de Grenoble (IGA - CERMOSEM) \\ Universidad de Sevilla (Departamento de geografia e historia)
}

\begin{abstract}
Résumé : Pour contribuer au développement du traitement géographique du phénomène musical, nous avons souhaité nous focaliser sur un objet particulier : le flamenco. En partant de ses caractéristiques propres, nous tenterons de faire le lien avec notre discipline dans une « géographie musicale flamenca ». Puis, pour avancer sur un terrain transdisciplinaire rassemblant géographie, urbanisme, économie et sciences politiques, nous verrons comment cet art peut être mobilisé comme une ressource territoriale. Dans cette mobilisation, les processus d'action collective seront particulièrement interrogés. Nous testerons donc l'hypothèse que l'aménagement du territoire en Andalousie se nourrirait, du moins sur le plan métaphorique, du bain culturel dans lequel se pratique le flamenco.
\end{abstract}

\begin{abstract}
To contribute to the development of the geographical treatment of musical phenomenon, we wished to focus one particular object: the flamenco. By leaving these appropriate characteristics, we shall try to make the link with our discipline in a "flamenco musical geography". Then, for advanced on a transdisciplinary ground, which take in count geography, urban planning, economy and political sciences, we shall see how this art can be mobilized as a territorial resource. In this mobilization, collective process will be interrogating. We argue so that the land settlement in Andalusia would so feed, at least on the metaphoric plan, of the cultural bath in which flamenco is practiced.
\end{abstract}

Resumen: Para contribuir al desarrollo del tratamiento geográfico del fenómeno musical, nos centraremos en el flamenco. Dada sus características propias, intentaremos relacionarlo con nuestra disciplina a través de una « geografía musical flamenca ». Entonces, para avanzar en un terreno transdisciplinario unificando geografía, economía, ciencias urbanísticas y políticas, veremos como este arte puede ser movilizado como un recurso territorial. Verificaremos aquí que la ordenación territorial en Andalucía se alimentaria pues, por lo menos en el plano metafórico, del baño cultural en el cual se practica el flamenco.

\section{Introduction}

Parce qu'il accuse à la fois une émancipation étendue à l'ensemble de la planète et une inscription locale forte, le flamenco et reconnu comme un phénomène musical hors norme. Par ce qui le lie aux préoccupations des sociétés contemporaines, par son inscription dans le temps passé et présent, Edgard Morin le qualifie « d'exemple médical » (France culture, 26 octobre 2008) quant à sa complexité systémique. Aussi, en Espagne, et plus précisément en Andalousie, les pratiques se multiplient, du tourisme global à l'expérience localisée, provocant à la fois l'émergence de confrontations culturelles, politiques et esthétiques, et l'accélération du rythme de fonctionnement des centres et moyens de création et de diffusion flamencologiques. Ainsi, les grandes scènes artistiques mondiales s'arrachent les artistes réputés, les écoles flamencas font le plein, l'industrie culturelle est fleurissante, fêtes et 
festivals sont au beau fixe. Pourtant, il semble que tous les acteurs du flamenco ne soient pas unanimes sur les effets positifs des transformations profondes du modèle flamenco. L'originalité et l'essence même de cet art semblent, selon certains, menacées par un système de plus en plus englobant qui en dénaturerait l'éthique et l'esthétique. Patrimonialisation, folklorisation et muséification issues notamment de sa mise en tourisme, en s'opposant aux schèmes de la créativité et de l'innovation, annoncent parfois une séparation. D'un côté, se trouve un flamenco fidèle à ses origines, pratiqué dans des cercles restreints, des lieux spécifiques, un flamenco du quotidien, un flamenco revendiqué principalement par une population gitane, un flamenco "puro »; de l'autre côté, un "pseudo-flamenco » (Lefranc, 1998), touristique et mercantilisé, vitrine de l'Andalousie culturelle, construit souvent ex nihilo par des artistes communs, poussé par le politique et l'économie de marché. Quelle réalité alors pour l'Andalousie flamenca ? Du modèle manichéen à celui de la complexité, comment le flamenco évolue-t-il vraiment dans la «glocalité » qui le détermine ? Quels lieux et quelles fonctions pour le flamenco dans cette société contemporaine ?

Pour apporter des éléments de réponse à cette problématique, nous tenterons de voir en quoi le flamenco est porteur d'un sens géographique. Pour ce, nous survolerons brièvement l'intérêt pour la géographie contemporaine de s'intéresser au phénomène musical. Ensuite, nous ferrons état des caractéristiques qui font du flamenco un objet fortement géographique. Puis, dans un élargissement interdisciplinaire de la question musicale aux sciences du territoire, nous tenterons de faire le lien avec la notion de ressource territoriale.

\section{La musique comme outil de lecture des territorialités émergentes}

Depuis les années 1990, un regain d'intérêt pour les faits de culture, initié dans le monde anglo-saxon, dynamise la recherche au travers de ce que l'on nomme couramment le « cultural turn » (Cook I. ; Crouch D. ; Naylor S. ; Ryan J., 2000). Ainsi, de nouveaux enjeux émergent des formes, objets et pratiques culturelles, abordés dans le sens restreint d'activités artistiques ou dans une version plus large englobant les modes de vie contemporains. Une lecture actualisée du monde est à la base de ces enjeux. Elle passe notamment par l'arrivée d'objets scientifiques insolites et de sujets novateurs. Elle entend, entre autre, prendre en compte les questions en marge, rejetées et oubliées des analyses traditionnelles, traiter notamment du banal et du quotidien, s'ouvrir à l'implicite et l'éphémère, donner sa chance au détail, à l'invisible, à l'immatériel. À l'intérieur de ce courant, la musique occupe une place non négligeable. Comme le montre la multiplication des travaux en géographie, une place incontestable lui est accordée dans la discipline, offrant aux géographes des pistes de recherches fécondes.

Du coté anglo-saxon, les premiers travaux (Peter Nash, 1968 ; Larry Ford, 1971 ; George Carney, 1974 et $1978 ; \ldots$ ) ont donné lieu à la multiplication d'initiatives pour déboucher sur un corpus aussi dense que varié, particulièrement depuis les années 2000. Ainsi, numéros spéciaux de revues géographiques (Anderson B., Morton F., Revill G., 2005; Waterman S., Brunn S., 2006;...) et ouvrages spécialisés (Bennett A. ; Hawkins S., 2004; Bandt R., Duffy M., MacKinnon D. (eds), 2007; Connell J.; Gibson C., 2003; Knight D.B., 2006; Krim A., 2007; ...) témoignent de cet engouement pour la thématique. Ici, les travaux américains et européens sont complétés par une riche production asiatique et océanique (Singapour, Nouvelles Zélande, Australie principalement). Si certaines des propositions restent en phase avec une géographie «classique », la grande variété de travaux s'ouvre aujourd'hui sur un vaste champ de possibles. Les articles programmatiques pour la recherche 
qui en ont fait état sont nombreux et aujourd'hui bien reconnus (Byklum, 1994 ; Kong, 1996 ; Leyshon, Matless \& Revill, 1998 ; Nash \& Carney, 1996 ; Smith, 2000).

En France, après quelques tentatives isolées en début de XXe siècle (Ladmirault, 1914; De Gironcourt, 1932) qui n'ont pas réussi à faire école, il faut attendre les années 1990 pour voir se structurer un mouvement collectif autour du sujet. Initiée (dans le cas de dynamique) à partir d'un mouvement abordant le sonore et ses liens multiples avec l'environnement, la musique prend une nouvelle place dans la géographie contemporaine via les travaux de Vauchey (1987) notamment. Ce précurseur en France, influencé par la géographie radicale anglo-saxonne, suit de près les travaux de Murray Shaffer tout en militant de son côté pour une qualité des diffusions radiophoniques. Puis, Jacques Lévy, aborde le sujet avec un article d'une revue musicale (1994) qui sera repris cinq ans plus tard dans Le tournant géographique (1999). On note également les avancées faites sur le terrain de l'opéra qui marquent le début des analyses situées en France (Foucher, 1996, puis Lamantia, 2005 ; Lacombe, 2007). Début 2000, une discussion aussi brève qu'intense montre que le sujet est porteur d'enjeux pour la discipline. Laurent Grison (2000) et Augustin Berque (2000) affichent ici une vision différente de la géographie, l'un trouvant des liens féconds entre musique et espace, l'autre lui rappelant que « acoustique n'est pas musique (et géométrie n'est pas géographie)». La discussion prend corps autour d'une conception de la géographie, " moderne classique » d'un coté, " post-moderne » de l'autre. La même année, Jean-Marie Romagnan (2000) signe un article phare dont la prétention sera de justifier la pertinence du sujet en ouvrant un large panel des possibilités de traitement. Il sera repris alors par une majorité des travaux postérieurs. A cela s'ajoute la publication ponctuelle de travaux aux sonorités de plus en plus disciplinaires, marquant l'introduction progressive de la musique dans la géographie française (Pailhé, 1998 et 2004 ; Apprill \& Dorier-Apprill, 1998 ; Raibaud, 2005).

Puis, avec la première journée scientifique affichée sur la thématique ${ }^{1}$, l'Université de Paris IV-Sorbonne marque en 2006 le début d'un mouvement qui résonne en $2007^{2}$, puis en $2009^{3}$, avec des perspectives de poursuite collective pour 2010 et $2011^{4}$. A Paris, un état des lieux des travaux, en France comme ailleurs, sert de loupe pour mieux observer les perspectives de développement de ce sous-courant disciplinaire (Guiu, 2006). Puis, l'Université de Bordeaux pose plus explicitement la question du lien entre musique et territoire en tentant de les rapprocher théoriquement et empiriquement (Raibaud (ed.), 2009). Finalement, c'est à Grenoble que le champ thématique s'élargit avec deux nouvelles entrées: une tentative d'explication de l'étonnement que crée l'annonce du sujet et les efforts de justification de sa pertinence qui en découlent et la mobilisation de la musique comme outil réflexif dans ce que nous appelons les «sciences du territoire $»^{5}$. Ici, la rencontre avec les anglophones Stanley Waterman et Michelle Duffy permettra aux géographes français de mieux s'essayer à l'international. Aussi, une certaine mise en cohérence des travaux est bien initiée. Elle passe autant par les événements scientifiques que par la production et la publication de travaux en commun. Les numéros spéciaux de revue se multiplient alors (Géocarrefour, 2003 ; Espaces

\footnotetext{
${ }^{1}$ « Musique et Géographie: quelles perspectives? », journée scientifique, Université Paris IV-Sorbonnes, 6 juin 2006.

${ }^{2}$ «Comment la musique vient au territoire? », Journée scientifique, Université de Bordeaux III, 12 mars 2007.

3 « Musique, territoire et développement local : Les Rencontres de Grenoble », Colloque International, Université de Grenoble, 19 et 20 novembre 2009.

${ }^{4}$ Deux rencontres, à Lyon en 2010, puis à Bordeaux en 2011 sont en cours de construction.

${ }^{5}$ Regroupant à Grenoble la géographie, l'aménagement du territoire, l'urbanisme, l'architecture, les sciences politiques, l'anthropologie et la philosophie autour de la thématique territoriale.
} 
et Sociétés, 2003 ; Culture et Géographie, 2006 ; Copyright Volume !, 2009 ; La Géographie, 2009).

Aussi, faire état des possibilités offertes par la multiplicité de géographes ayant travaillé sur la musique ne peut avoir sa place ici. D'abord, ce travail à déjà été fait au fur et à mesure que le sous-courant disciplinaire s'est affirmé en géographie, tant du côté anglophone (Carney \& Nash, 1996 ; Kong, 1995 ; Smith, 1994) que francophone (Guiu, 2009). Ensuite, la riche production bibliographique est révélatrice d'une multitude de possibles qui touchent aujourd'hui la quasi-totalité des thématiques qu'offre la géographie sociale et culturelle contemporaine. En faire une liste intelligible serait couteux en place et relève plutôt d'un travail de doctorat. Finalement, notre volonté est d'apporter, avec cette proposition ponctuelle, une réflexion concernant les quelques vides qui restent à combler dans ce corpus, en se penchant notamment sur la notion de ressource territoriale. Dans l'optique de poursuivre la recherche des possibilités de traitement de la musique comme objet spatial et territorial, nous avons donc souhaité étudier l'exemple du flamenco. Cette recherche, plutôt que d'attaquer le versant épistémologique de la discipline, tente de dresser un horizon géographique de l'art pour contribuer au tournant que la discipline opère. Après une introduction à la spatialité du flamenco, nous nous proposons d'en interroger ses dimensions identitaires pour opérer ainsi un glissement de l'espace au territoire. Nous avons ensuite rapporté l'objet à son intégration dans les politiques de développement territorial. L'exemple de la ville de Jerez de la Frontera (Xérès - Espagne) tentera ainsi d'enrichir les recherches portant sur les nouveaux dispositifs d'action collective.

\section{Le flamenco : un objet géographique}

En se positionnant pour une indispensable intrication des sources explicatives, nous pensons qu'un point de départ acceptable serait celui d'une histoire pour la géographie du flamenco, au même titre qu'une géographie pour l'histoire de celui-ci. Comme l'affirme Cristina Cruces Ròldan, "il ne peut y avoir de séparation entre le flamenco, son histoire et sa géographie" (Cruces Roldan, 2002). D'abord parce que, selon Pierre Lefranc (1998), l'essentiel de l'héritage du flamenco que représente le cante jondo "a été crée et cultivé à l'intérieur de trois ou quatre douzaines de familles gitanes sédentarisées depuis des siècles dans une région très limitée de Basse Andalousie [...] la région est située [...] dans un triangle Cadiz/Jerez de la Frontera/Triana.". Sa localisation, donc précisément située, est le fruit de la relation entre un peuple, une terre et leurs histoires relatives. Ensuite, parce que ses modes de production et de diffusion ainsi que leur évolution dans le temps sont à la source de ce qu'est le flamenco aujourd'hui. Cet art est donc localisé et localisable en des lieux précis, par ses origines puis ses modes de diffusion; ce qui justifie pour nous la pertinence d'une "géographie du flamenco" ; mais surtout, ces mêmes lieux pourraient être à la base de sa territorialisation. Aussi, comment expliquer cette difficulté qu'a longtemps rencontrée le flamenco à être écouté et compris en dehors des frontières andalouses ? Comment considérer son émancipation fulgurante en Espagne, puis dans le reste du monde ? Le caractère endémique mis en valeur par les acteurs du flamenco est alors très présent dans les textes relatant cette culture. Pour en comprendre les mécanismes actuels, il nous faudra donc passer par les processus d'émergence, de construction et de diffusion, aussi bref en soit le détour.

Le flamenco, tel qu'on le connaît de nos jours, est, semble-t-il, une manifestation artistique qui commence à jouer un rôle social fort à partir du XVIIIe siècle. Il s'exprime d'abord dans les lieux de travail paysans et ouvriers ou dans les réunions familiales de l'ethnie 
gitane principalement, où se construisent les bases d'un chant dont les origines profondes restent encore un mystère. Les gitans et les paysans en furent les principaux interprètes, car la société " cultivée » et élitiste le méprisait, le considérant comme affaire de petites gens. Cette attitude favorisa le contact entre les gitans et le peuple andalou. C'est depuis lors que cet art marginal tendra à s'insérer dans la société, d'abord dans les classes populaires andalouses, puis peu à peu dans toutes les classes sociales. Viendra ensuite le tournant de "l'époque hermétique du cante" (Sandoval, 1998) se situant dans la seconde moitié du XIXe siècle, grâce à la création des "cafes cantantes" ${ }^{\prime 6}$ et à la professionnalisation des artistes. Enfin, se développera peu à peu ce qui fit en partie le succès contemporain de la discipline : les festivals en plein air, dont la plupart combinent, encore aujourd'hui, musique et gastronomie régionale : el Gazpacho de Moròn, la Caracolà de Lebrija et el Potaje de Utrera; ainsi que les « joutes flamencas », notamment dans les grandes villes. Cette première mise en rapport du flamenco s'explique alors par la relation entre espace privé et espace public, et le rôle qu'ils jouent respectivement dans la sociabilité du flamenco. Elle est exprimée de la manière suivante par Cristina Cruces Roldan (Cf. Tableau ${ }^{\circ} 1$ ) qui sépare les espaces privés comme appartenant à un «flamenco d'usage » et les espaces publics à un «flamenco du changement ».

\footnotetext{
${ }^{6}$ « Cafés chantants », lieux de mise en publique du flamenco.
} 
Tableau $n^{\circ} 1$ : les espaces du flamenco

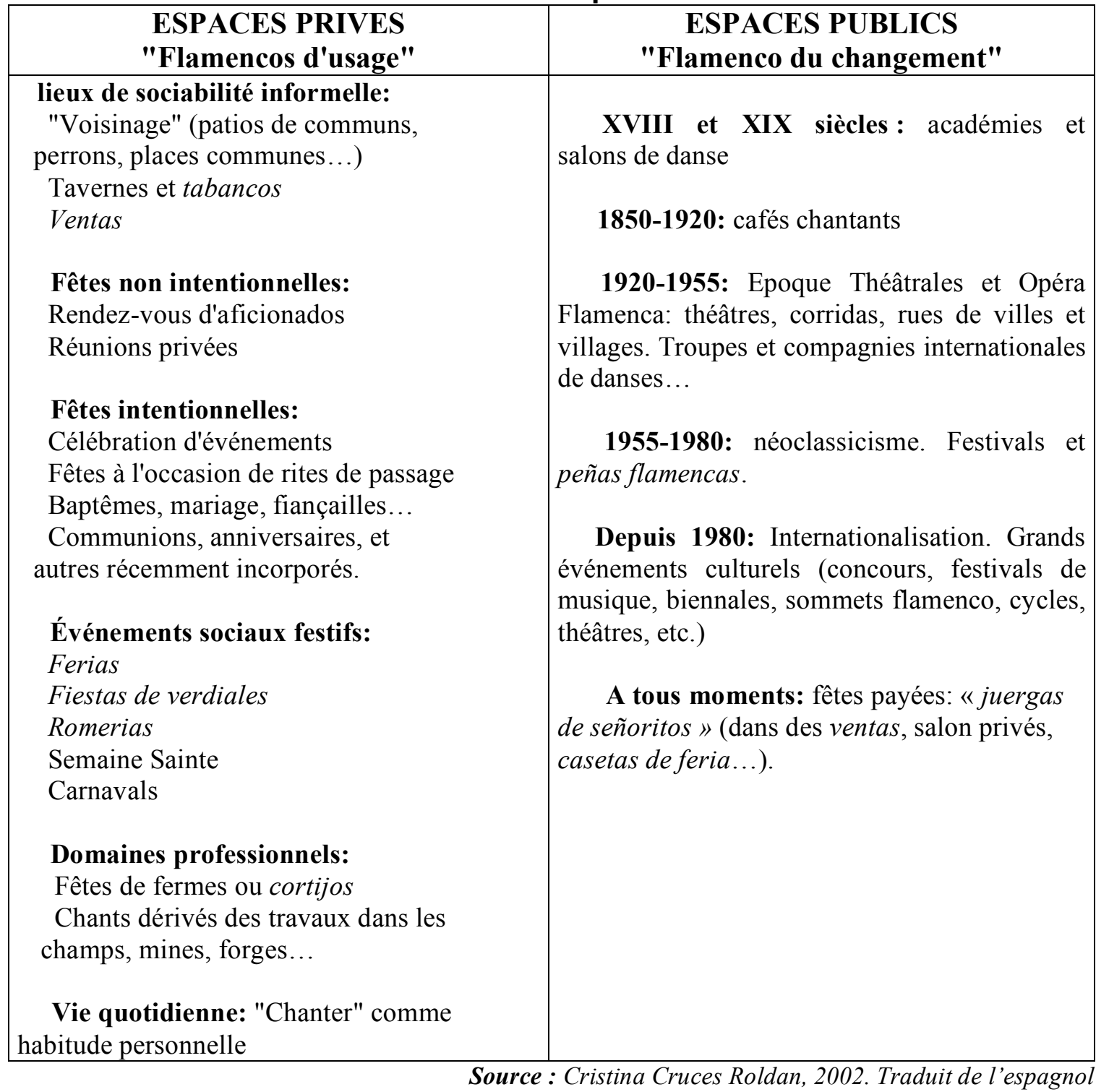

Il semble en effet que ce soit cette mise en public, aux travers de différents lieux, qui ait contribué à la modification des formes du flamenco. Un flamenco populaire, plus « audible » plus joyeux aussi, est alors développé au détriment parfois de l'utilité cathartique et libératrice d'un chant profond pratiqué dans un cercle restreint en espace privé ; (le cante jondo, et particulièrement a palo seco, c'est à dire a capella, qui constitue la base historique du flamenco, étant souvent décrit par les auditeurs non-avertis comme « inaudible »). Ainsi, la meilleure accessibilité physique et esthétique va rapidement démultiplier le public, et par làmême le nombre de pratiquants.

Nous devons toutefois préciser que l'opposition espace privé/espace public se doit d'être relativisée. S'il existe effectivement une relation dialectique entre deux flamencos en fonction de leur espace de pratique, la diffusion de cet art se doit d'être abordée sur un plan multilatéral, les interrelations entre artistes et public n'étant pas toujours clairement définies. Par exemple, le «monde familial» se retrouve aussi dans les prestations en public, les artistes étant très généralement accompagnés de leur peña, qui les encourage dans un jaleo plus ou moins imposant. De même, le flamenco chanté pendant la semaine sainte ou les 
carnavals l'est dans un espace semi-privé où le public extérieur joue un rôle d'auditeur-acteur, même s'il n'influe pas sur les conditions de production, notamment par le biais financier. Ce serait donc la question transversale d'ambiance qui jouerait également un rôle dans la construction du flamenco, est serait alors le principal actant de l'apparition du duende.

Aussi, c'est principalement leur rapport à la géographie qui caractérisera peu à peu les différents flamencos. Nous souhaitons donc insister, sans pour autant nous confiner dans une approche déterministe, sur la localisation des différentes ramifications du flamenco, et sur l'importance de celles-ci dans la construction du processus de diffusion. "Le flamenco fut et est [...] un fait culturel inexplicable en dehors des limites de l'Andalousie, de sa réalité sociale, économique et culturelle de ces deux derniers siècles, qui fut le temps pendant lequel il s'est structuré et consolidé pour atteindre les modes d'expression que nous lui connaissons, pour autant qu'il trouve de nos jours des centres et des lieux extra-andalous dans lesquels il se développe de manière notable" (Suárez Japón, Op.Cit.). Les différents palos (une cinquantaine environ: buleria, taranto, tango, soleares...divisés eux même en différents sous-styles, difficilement nombrables) ont des origines géographiquement situées, la plupart du temps relatives à la ville où ils sont apparus, ou encore liées aux milieux culturels et socioprofessionnels auxquels ils appartiennent. Par exemple, la rondeña est le palos originaire de la ville de Ronda, la buléria attribué à la région de Jerez, le fandango plutôt caractéristique de Huelva, etc. Sur le même mode, le martinete est un chant originaire des forges, accompagné uniquement $\mathrm{du}$ son du marteau frappant l'enclume, le cante de las minas originaire des mines, etc. Cette "géographie musicale" qui passe par la multitude de palos et l'importance de leur provenance se doit alors d'être doublée de leur relation aux interprètes qui les mobilisent. Même si aujourd'hui les répertoires sont de plus en plus mixtes (ce qui pourrait en partie contribuer à la déterritorialisation du flamenco ${ }^{7}$ ), chaque cantaor interprète régulièrement une variété réduite de palos propre à son origine géographique. De plus, les noms de scène des artistes, souvent attribués par les proches et le public, sont très souvent porteurs de cette même provenance (Camaròn de la Isla, El Capullo de Jerez, Bernada de Utrera, Béni de Cadiz,...). C'est finalement au niveau de la toponymie singulière du flamenco que la géographie agit directement, au travers de ce que Antonio Caffarena (1964) nomma les " ciudades cantaora » ("villes chanteuses »), centres névralgiques où furent développées les différentes spécificités de cet art et d'où viennent la plupart des grands interprètes, ainsi que des «comarcas cantaoras», qui correspondent à « l'espace plus large qu'elles [les ciudades cantaoras] ont centralisé » (Suarez Japon, 2002). Plus localement, ce sont les quartiers urbains qui ont joué un grand rôle dans la différenciation des flamencos en fonction de leur géographie. Pour ne prendre qu'un exemple fort, le quartier sévillan de Triana, sans aucun doute un lieu mythique du flamenco, est originellement séparé par le fleuve Guadalquivir du centre historique de la ville. Cette opposition rive droite/rive gauche fut à l'origine d'une ségrégation ethnique entre gitans et payos (principalement due à l'absence de pont, puis de pont fixe) qui, bien qu'aujourd'hui estompée, participa de la création un terreau fertile à l'émancipation d'un flamenco caractéristique. Il peut également exister des différences entre des localités proches tels des quartiers urbains (Santiago et San Miguel à Jerez, par exemple), ou encore des emboîtements d'échelles (p.e. cercle familial, quartier, ville, comarca pour une même palo) qui seront différenciés par la variation de estilos ou de soniquetes. C'est ainsi que le rôle de la ville andalouse et la manière dont s'est organisé son espace se doit d'être pris en compte lors des tentatives d'explication du paysage musical actuel. Juan Manuel Suarez Japon (2002), par analogie avec le modèle du géographe Georges Bertrand (1968), détermine

\footnotetext{
${ }^{7}$ Francisco Ollero Obato, entretien personnel, Séville le 19 février 2008.
} 
ainsi la maison flamenca comme " géotope du flamenco », unité d'échelle mineure, le quartier comme "géofaciès», échelle intermédiaire d'analyse, et les «agrovilles andalouses» représenteraient un "géosystème du flamenco ». Finalement, il est tenté de qualifier la situation andalouse du flamenco de « climax ».

Il est donc évident que l'approche spatiale et régionale du flamenco comme phénomène musical est indispensable à sa bonne compréhension, car " c'est la dimension territoriale qui détermine qu'un fait quelconque puisse être analysé géographiquement, et le flamenco est une manifestation génétiquement andalouse, inscription qui ne fait aucun doute à la lumière de l'analyse historique et culturelle » (Manfredi D., 1955). Mais, la question andalouse doit être relativisée. D'abord, par le simple fait que les régions de Murcie et d'Estrémadure, particulièrement, ont elles aussi accueilli des artistes éminents dès l'apparition du flamenco. Ensuite, par le confortement de Madrid et de Barcelone (ainsi qu'une partie du sud-est de la France, qualifiée pour l'occasion de «nouvelle Andalousie musicale » (Fanise P., 2002.)) comme lieux dynamiques de création, de production et de diffusion. Aussi, pour la présence de plus en plus forte de spectacles d'excellence en dehors de cette région (reste de l'Espagne, France, Amérique et Japon entre autres). Enfin, il existe de nombreuses interrelations entre le flamenco et d'autres influences musicales dont, en plus des liens avec la musique arabo-andalouse et le répertoire traditionnel andalou et espagnol, l'exemple des cantes de ida y vuelta (littéralement : "chants d'aller et de retour"), hybridation musicale entre le flamenco, des répertoires espagnols et les musiques d'Amérique latine, est certainement le plus marquant.

Par ces différents aspects, l'analyse géographique du flamenco a des similarités avec celle du tango, notamment pour le rapport local/global qui en détermine la dynamique. S'il « est né quelque part avant de naître à une époque » (Apprill C. ; Dorier Apprill E., 1998), les unités de lieux et de temps qui le caractérisent sont aujourd'hui plus que jamais entremêlées. Les différents flamencos appuient cette nécessaire explication par l'histoire et la géographie. Les « sentiers de dépendance » jouant un rôle majeur dans la compréhension de l'état actuel du phénomène. La globalisation $a$, par exemple, permis de multiplier les moyens de diffusion de cet art, et par là même ceux de production. Ainsi, le flamenco a su s'exporter en Europe et en Amérique du Nord ainsi qu'au Maghreb, mais aussi dans d'autres pays moins attendus comme le Japon, en Bosnie ou en Afrique du Sud par exemple. Il est alors devenu courant, voir même une dominante dans certaines écoles flamencas sévillanes, de voir des japonais(es) apprendre et reproduire un flamenco parfois des plus traditionnels. Des grands festivals ont lieu, de Tokyo à New York, de Mont-de-Marsan à Düsseldorf, dans un espace mondial criblé de lieux où le flux artistique du duende construit le réseau des territorialités flamencas multiples. Ainsi, en partant d'une l'analyse spatiale du flamenco, les conditions de sa construction d'objet géographique nous ouvrent la recherche à sa dimension territoriale.

\section{Du produit identitaire à la ressource territoriale}

Pour aborder alors le flamenco avec un des concepts émergents dans les sciences géographiques ouvertes au concept de territoire, nous nous proposons d'opérer un croisement avec la notion de ressource territoriale. Cette notion, retravaillée récemment, "s'impose comme une figure nouvelle du développement et de l'aménagement des économies dans le grand bouleversement post fordiste de la mondialisation » (Gumuchian ; Pecqueur, 2007, p.5). Les caractères fondamentaux que ces auteurs donnent à la ressource territoriale semblent se retrouver alors dans le flamenco: un attribut de position marqué par une construction 
historique et géographique, ce que nous venons de considérer ; la constructibilité en lien avec la dynamique territoriale, notamment en terme de patrimonialisation, démonstration à laquelle nous aspirons ; la complexité systémique, affirmée plus haut avec la citation de Morin ; et la question du sens même de la notion dont nous espérons participer à la construction. Le rapprochement entre les aspects de produit, de patrimoine et de ressource du flamenco permis par la démonstration de son inscription territoriale est à l'origine de cette construction. En passant par le tourisme culturel, nous élargirons le débat au rôle des politiques dans ce processus d'émergence de nouvelles territorialités flamencas, afin de proposer une approche analytique de cet art au travers de son injonction dans l'économie locale. À partir de l'observation de l'appropriation et de l'instrumentalisation de la culture flamenca par une partie de la société andalouse, nous attirerons finalement l'attention sur le fait que le fruit de son expression métaphorique peut conduire, dans le cas de la ville de Jerez-de-la-Frontera, à l'inflexion de la pensée planificatrice.

Aujourd'hui, le niveau de reconnaissance sociale du flamenco est à son comble, à tel point que l'on commence à le placer au même rang que la musique classique, pour l'importance patrimoniale qu'il représente; il est devenu une véritable institution, donnant lieux à la deuxième chair de musicologie du monde occidental dédiée à un seul style musical : la flamencologie. Aussi, ce qui frappe aujourd'hui ses acteurs, c'est sa capacité à s'exporter d'une culture à l'autre, son caractère international, et surtout les adaptations qui en découlent, pas toujours placées sous le regard positif des défenseurs d'un flamenco fidèle à ses origines. Ce constat semble être à la base du processus de patrimonialisation.

« En quelques décennies, le flamenco est passé d'une manifestation culturelle ignorée et sous valorisée, à une position de privilégié dans les politiques culturelles de la communauté autonome andalouse. Il s'est alors converti en un des plus forts marqueurs identitaires de cette collectivité » (Vega Lopez J., 2007). Sachant que sa transmission s'est faite le plus souvent de manière orale, par contact direct entre artistes et entre artistes et publics, il s'est formé un « caractère héréditaire dynastique » propre à quelques micro groupes, il a également été défini comme une "manifestation individuelle par excellence où l'individu guide le groupe» (Grimaldos A., 1998). Or, sa patrimonialisation propose d'en faire un bien culturel commun, un objet collectivement partagé, un « marqueur d'identité culturelle ». Nous sommes ici face à son activation en tant que ressource touristique. Ce mouvement général d'appropriation du flamenco, dans ses origines, mais surtout dans sa forme actuelle, tend à provoquer des positions conflictuelles entre les différents acteurs. D'un côté, un large programme de patrimonialisation, orchestré politiquement au niveau régional, par la Junta de Andalucia, et au niveau national, par certaines actions du gouvernement espagnol ou d'autres Régions, oscille entre une lecture holistique visant le bénéfice économique et socioculturel que pourrait apporter cette opération et le risque de fixation et de déperdition des valeurs fondamentales d'un flamenco pluriel. De l'autre, de manière plus sporadique, au niveau provincial, local, " sous-local » comme le nomment certains, et même parfois familial, est revendiquée une expression artistique unique pour chacun, mais néanmoins un bien universel pour l'humanité. Le flamenco se manifeste alors sous de multiples formes: pratiques privées, spectacles, industrie du disque, cours et études, lutherie, haute couture et mode vestimentaire..., qui constituent également des ressources pour la société andalouse. Mais, géré comme une ressource patrimoniale, le flamenco fait l'objet malgré lui d'une double mutation : artistique et symbolique.

D'abord, parce qu'il peut être considéré comme une simple activité culturelle et artistique qui rassemble musique, danse et chant, alors qu'il correspond également, pour toute une frange de la population, à un véritable mode de vie. Ensuite, parce que ce mode de vie est 
aujourd'hui atteint dans ses fonctionnements, par les processus englobants de mondialisation et de métropolisation. Il est réapproprié à des fins politiques et économiques, voire mercantiles, par les pouvoirs locaux qui feintaient de l'ignorer quelques dizaines d'années auparavant. Cela n'est donc pas sans créer des effets pervers: confrontations administration/administrés, gitans/non-gitans, andalous/espagnols, perte de la «diversité flamenca », création ex nihilo d' " authenticité » et de "pureté », projets urbains et touristiques démesurés...Est-il alors possible de conserver l'idée d'une ressource territoriale dont les modes de mobilisation ne fassent pas consensus chez une partie des acteurs principalement concernés? Cette même mobilisation ne constitue-t-elle pas un "risque socioculturel" ${ }^{8}$, particulièrement dans un contexte où l'homogénéisation culturelle crée craintes et incertitudes ? La complexité même du flamenco, dans ses différentes acceptions comme dans ses caractéristiques territoriales et son "ultra localité", n'empêcherait-elle pas sa mobilisation commune et unanime par les territoires, aussi réduits soient-ils ?

Politiquement, le flamenco fut « oublié des administrations jusqu'au début des années $1990 »$ (Guiguère H., 2005a). Cela ne fait en effet que peu de temps que les autorités andalouses et espagnoles considèrent le flamenco comme une "possibilité de capitalisation politique et mercantile » (Guiguère H., 2005b). Un point de départ se constitua autour de la construction d'un patrimoine commun par des initiatives publiques et ce que beaucoup décrivent comme «l'invention du passé flamenco ». Pour Gerhard Steingress (2002:45), qui postule pour " une construction artificielle de l'identité andalouse », " nous sommes alors devant un phénomène d'idéologisation de la culture en fonction du rétablissement de bases de la cohésion sociale au travers de la conscience collective dans une société désidéologisée et sécularisée ou, dit autrement, devant un concept neo-durkheimien ». La patrimonialisation dont le flamenco fait l'objet est en effet critiquée sur différents points. D'abord, elle freinerait la dynamique propre à ce dernier en le folklorisant et en le «muséifiant». Ensuite, elle rendrait commune une histoire et une culture qui n'est en aucun cas la même pour chacun ni revendiquée par tous les andalous. Enfin, elle tendrait à simplifier la complexité du phénomène, comme sa mise en tourisme avant elle, en en vulgarisant ses formes et expressions pour se rapprocher d'un art plus accessible.

«Le flamenco rencontre un domaine de développement et de fleurissement dans les petites villes en privé, dans la solitude du travail de la forge, du champ ou de la prison, par exemple, qui sert sa liberté d'exprimer ses sentiments les plus profonds » (Mairena A., 1976). Aussi, dans le flamenco, beaucoup se référent à la terre, et défendent ainsi une certaine « appellation d'origine contrôlée » (Macías, 2007) pour marquer une singularité, un sentiment d'identité, mais aussi pour prétendre à une légitimité que d'autres n'auraient pas. Or, cette expression du local proviendrait surtout, pour les raisons actuelles dérivées des explications historiques abordées plus haut, en plus des artistes et professeurs payos, des acteurs politiques impliqués dans la gestion de cet art. Les gitans, eux, tendraient plutôt à faire référence au sang, à la filiation familiale et à l'hérédité. Pour ces raisons notamment, " quelques grandes

\footnotetext{
8 Nous nous détachons bien évidemment de la définition originale de «risque culturel», pour définir une typologie de risque (dangerosité + vulnérabilité) dont l'impact serait socioculturel (dispersion des valeurs citoyenne, conflit ethnique et sociaux, hypercommercialisation et effacement de l'éthique artistique, désengagement collectif, ...) ; voir Canova N., « Du développement des territoires culturels au développement culturel des territoires », Colloque international "Le développement culturel: un avenir pour les territoires ?", 1718 avril 2008, Université de Nîmes et N. Canova, "Questionner les risques de la mobilisation du patrimoine culturel immatériel dans le développement territorial», communication aux 2nd journée nationales du patrimoine culturel immatériel, 27 et28 novembre 2009, Corum St Jean, Clermont-Ferrand.
} 
familles gitanes tendent à rentrer en conflit avec l'administration qui persiste dans la diffusion du flamenco comme produit touristique et mercantile » (Guiguère H., 2005b).

Une autre problématique actuelle tourne autour de la «dépersonnalisation» du flamenco par une surévaluation du local et de l'importance de cette localisation à des fins artistiques ou emblématiques. Et ce à tel point qu'aujourd'hui, nombre d'acteurs du flamenco militent pour une nouvelle appellation: celle d'un "art gitan » plutôt que celle "d'art andalou ». Pourtant, Gerhard Steingress (2002), tout en s'opposant à la définition "d'art total » donnée par Cristina Cruces (2002), souligne les éventualités d'effets pervers issus d'une ethnicisation du flamenco, en citant le diplomate et écrivain José María Ridao (« ...le risque que courent les sociétés contemporaines si elles se définissent "en terme d'identité » au lieu "de citoyenneté. ») puis en proposant un regard sur les travaux d'Anthony Giddens (1991) notamment : " le pouvoir est toujours incorporé aux traditions, parce que l'identité collective tend à être "une construction sociale produite par des groupes sociaux porteurs qui actent dans des situations particulières et en fonction d'une charpente de code symbolique » (Steingress, op.cit., :49). Il nous faut donc rappeler que le flamenco n'est pas plus un art spécifiquement gitan dans ses origines qu'il n'est andalou aujourd'hui, même si cela contribue à le déterminer.

Les pouvoirs publics locaux qui tendent à mobiliser le dynamisme des activités traditionnelles en les centralisant et en essayant d'en contrôler le développement sembleraient alors être à l'origine de cette discorde. La récente polémique sur « la compétence exclusive » en matière de flamenco, déclarée par l'article 67 du nouveau Statut de la Région Andalousie et qui a suscité bien des critiques tant de la part des autres régions où existe une fibre flamenca qu'à l'intérieur même de l'Andalousie, appuie la dimension politique et stratégique que révèle cette néo-patrimonialisation. Le flamenco a traditionnellement été traité au niveau touristique comme «complément et subordination à d'autres produits touristiques dans une perspective principalement folklorique qui a certainement contribué à son immobilisme et à une perte de qualité dans certains milieux » (Deval, 1989). Aussi, nombreux sont les contacts qui affirment que les stratégies mercantilistes des grandes entreprises de discographie et de communication ont contribué au développement d'un « risque socioculturel ${ }^{9}$, mettant alors en danger "la survie de son authenticité et pureté » (Martín Martín M., 1995), de par l'absence de connaissances de ses valeurs anthropologiques et musicologiques notamment. Aussi, il «n'est pas clair que le flamenco soit un "marqueur d'identité culturelle, ni ne se révèle un véritable contenu de son caractère populaire, ni recommandable sa soumission à la stratégie d'un nationalisme andalou sous-jacent, camouflé derrière une certainement très problématique acception de «patrimoine culturel »» (Steingress, op.cit.).

A Jerez de la Frontera, où $15 \%$ de la population est gitane, il semble qu'il y a une différence entre le discours ambiant sur l'intégration des gitans et la réalité. La ville qui prétend «développer et conserver la culture flamenca» ne semble pas pour autant se préoccuper de l'exclusion et de la marginalisation dont souffrent encore nombre d'entre eux, pas plus que de la disparition progressive du Kalé (parler gitan, souvent utilisé dans le flamenco). "Ils devraient le faire eux même », répond ainsi la chargée de communication aux questions d'Hélène Guiguert (2005a), concernant cette non-action de la mairie. Ainsi, cette dialectique administration/administrés, relativement forte en Andalousie, tend à vouloir s'effacer par le discours. Dès lors, le flamenco est établi comme source régionale de richesse économique, mais aussi socio-cuturelle et constitue çà et là un élément fondateur des

\footnotetext{
${ }^{9} 28$ novembre 2009, N. Canova, « Questionner les risques de la mobilisation du patrimoine culturel immatériel dans le développement territorial », communication aux $2^{\text {nd }}$ journée nationales du patrimoine culturel immatériel, 27 et28 novembre 2009, Corum St Jean, Clermont-Ferrand.
} 
programmes de développement souhaité «durable». Mais, se pose alors la question "des villes ou peuples qui auraient les atouts nécessaires pour élaborer un modèle de développement touristique basé sur des principes de protection et de soutenabilité ${ }^{10}$. Le consensus difficile à établir ici semble se refermer sur les arguments suivants : d'abord une reconnaissance au niveau international comme un haut-lieu du flamenco, ensuite sur les espaces qui contribuent à la production d'une identité flamenca : "maisons familiales, patios de voisinages, quartiers populaires, tavernes et bars, et autres espaces urbains. " (Molina R., Espìn M., 1992). Viennent enfin les infrastructures relatives au flamenco: (toujours pour l'exemple de Jerez de la Frontera) Centre Andalou de Flamenco, Institut de Flamencologie de Jerez, antenne du Département de Flamencologie de l'Université de Cadix, Délégation de la Culture de la Mairie rattachée au Ministère de la Culture, Fondation de Théâtre Villamarta, 16 peñas flamencas, 11 écoles de danse et de guitare, sans compter tous les événements (festivals, concours, fêtes, séminaires...) ainsi que les initiatives locales et activités non répertoriées. Tout cet « arsenal » flamenco contribue à la création d'activités et d'emplois sur la ville, pour et par la population locale. Il relève d'une politique de coordination et de planification qui configure, promeut et ajuste ce «produit» dans l'ensemble de l'offre touristique globale de Xérès. Pourtant, les aberrations de l'administration n'en sont pas moins réelles.

L'exemple de la « ciudad del flamenco », tout en illustrant le fait que le flamenco, plus que de posséder une grande potentialité touristique autochtone, puisse constituer une véritable ressource locale de dynamisme économique et socioculturel certes, mais aussi urbanistique, marque l'enjeu politique que peut constituer sa reterritorialisation. La «Ciudad del flamenco est une des actions urbanistiques les plus ambitieuses de Xérès » affirmait Pedro Pacheco Herrera, ancien maire à l'origine du projet de cette commune d'environ 200000 habitants. Ce projet, alors en processus de reconfiguration, doit prendre place dans des espaces et bâtiments de la Plaza de Belén, au cœur de la ville médiévale et du centre de l'ancienne médina, "point équidistant entre les quartiers les plus flamencos de Xérès: Santiago et San Miguel ». Présenté comme un complexe culturel pour la promotion et la divulgation de cet art, il devait initialement compter avec un auditorium national et des salles de répétitions annexes, une Ecole Supérieure d'Art Flamenco divisée en deux départements: apprentissage et perfectionnement, un centre de recherche et de documentation, un musée, ainsi qu'un Centre Européen de Musique Andalousite. Il a fait l'objet d'un concours international d'architecture en 2003, que la proposition des architectes suisses Jacques Herzog et Pierre De Meuron remporta à l'unanimité en 2004. Cette proposition (Herzog et De Meuron, 2004). se base sur un jardin comme point de départ, illustrant alors la terre andalouse comme symbole de la naissance du flamenco, et propose une élimination des « contraintes architectonico-créatives » par l'englobement de plusieurs édifices, existants ou nouveaux, en « une ville dans la ville ». Ainsi, ce « puzzle de fragments » sera capable de croître dans une construction incrémentale du projet, au grès des nécessités, des changements, des humeurs de la ville; à l'image des prestations flamencas. Une tour, rappelant l'ancienne médina " dialoguera avec celle de l'Alcazar et de la cathédrale », comme dans un échange entre cantaor et guitariste. Un " jeu intérieur/extérieur », avec notamment un mur perforé comme enceinte, rappelle l'importance des médiations entre espace public et espace privé pour le flamenco. Cette proposition peut être entendue comme «une topographie artificielle, iconographique, enracinée dans la tradition flamenca ». Le modèle de planification urbanistique projeté prend forme directement autour du flamenco. D'abord directement,

\footnotetext{
${ }^{10}$ Mariano Ruiz Carretero, économiste, Université de Jerez de la Frontera.
} 
puisqu'il est la raison d'être du projet, mais aussi idéologiquement, car une pensée flamenca est traduite dans la proposition et est certainement à la base de la décision finale. La Ciudad del flamenco est également présentée comme une opportunité de régénération et de développement urbain. "Elle est le point central des opérations projetées par le département d'urbanisme de Xérès » (Diario de Jerez, 20 septembre 2005). Le quartier dans lequel elle prend forme est, malgré sa position hypercentrique, en réelle décomposition. Les activités qui vont se développer ici seront donc une aubaine pour le quartier, qui attend depuis longtemps son " tour » dans le programme de rénovation urbaine : des bâtiments nouveaux et d'autres réhabilités, des emplois directs et indirects, et surtout une population nouvelle de visiteurs, du touriste « classique » au haut fonctionnaire européen, de l'élève débutant à l'artiste célèbre, de l'aventurier curieux au flamencologue averti.

Nous voyons donc, de manière plus ou moins évidente, comment les pouvoirs publics d'abord, mais aussi l'ensemble des acteurs de cette "ville du flamenco », se saisissent des principes et de la pensée que véhicule le flamenco. En reliant une pratique culturelle avec l'action urbanistique par le biais métaphorique, le résultat hybride se rapproche alors de ce que nous nommerons une "pensée planificatrice flamenca». Les conditions dans lesquelles évolue la pensée aménagiste, ce contexte flamenco qui l'entoure et percole, laissent supposer que sa construction est continuellement infléchie, influencée, détournée, soulevée et finalement enrichie par les traces plus ou moins visibles que laisse la culture locale. Ce qui nous permet finalement de faire le lien, du moins sur le versant pragmatique, avec les travaux que porte Olivier Soubeyran sur la « métaphore musicale et la pensée aménagiste».

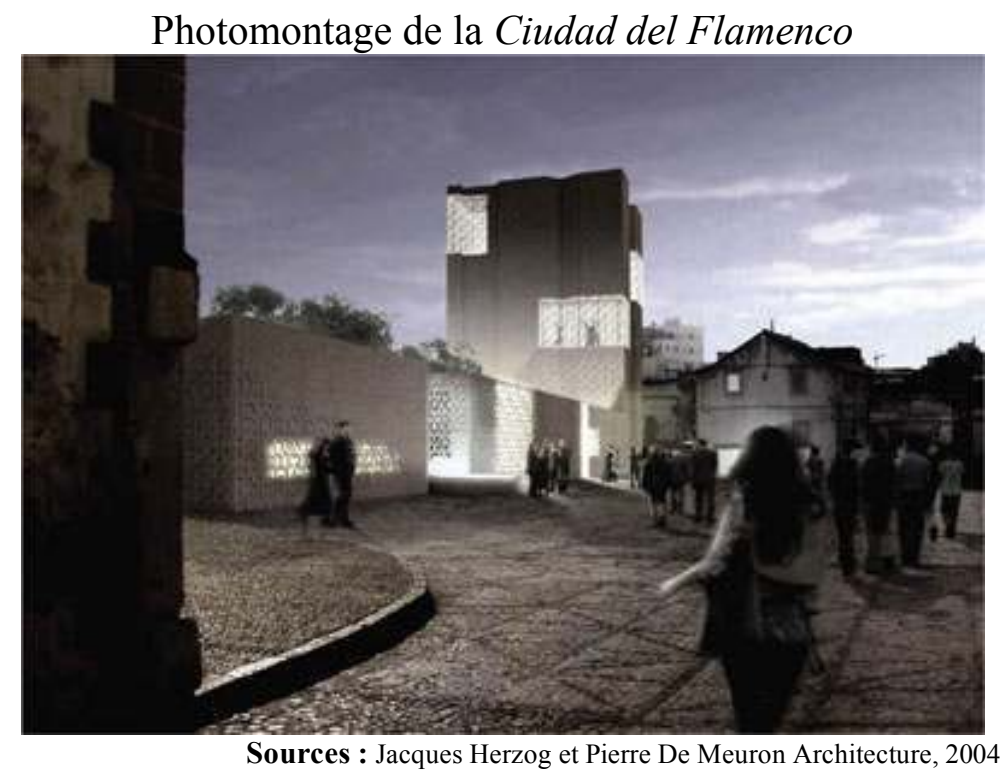

Etat actuel de la Plaza de Belén 


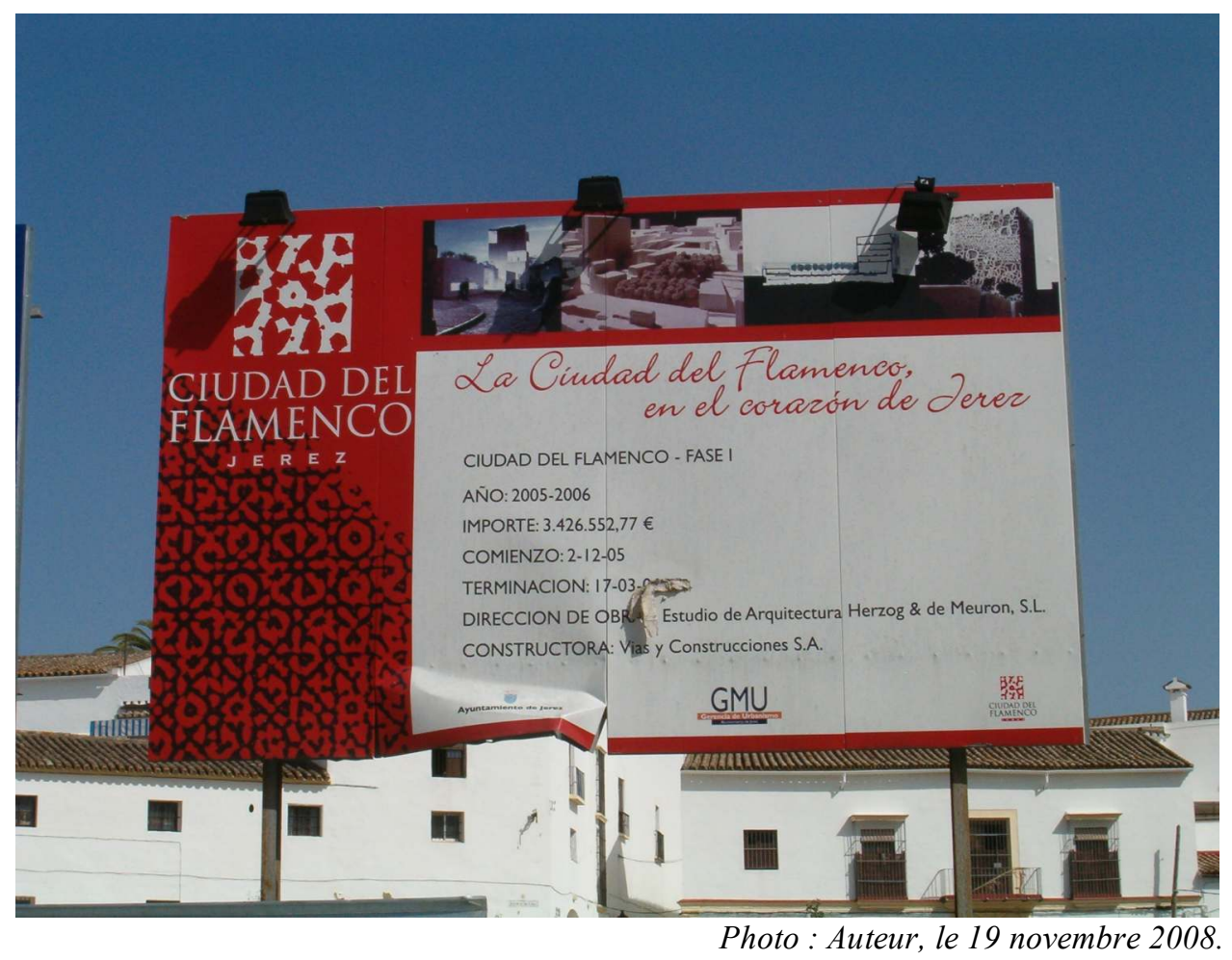

Cependant, dans cette région où le clivage gauche/droite est majeur en termes d'action collective, l'arrivée au pouvoir d'une maire socialiste, Pilar Sánchez Muñoz, vient supporter les opposants d'un projet critiqué en certains points. Par exemple, l'architecture mauresque est mise en avant alors qu'elle n'est pas particulièrement représentative de l'Andalousie populaire, gitane et flamenca. De même, l'absence d'une représentation gitane dans le Conseil d'Administration reste gênante lorsque nombre de gérants de la municipalité disent ouvertement que «le flamenco appartient au gitan» (Guiguert H., 2002a). Aussi, pour ce projet initialement prévu de 3.500.000 euro, et dans lequel ont déjà été dépensés 10 millions rien qu'en cimentation, les architectes n'auraient pas su conserver l'idée d'une construction incrémentale, fonction des moyens disponibles et des réajustements souhaités. Ils n'avaient par exemple pas prévu les contraintes que le sol local, à la fois extrêmement malléable et riche de trouvailles archéologiques, allait poser.

Finalement, un frein politique complète les manques. La municipalité de Jerez (tenue par le Partido Popular) semble en effet n'avoir pas su consulter la Région Andalousie (historiquement tenue par le $P S O E$ ) et ses partenaires quant au développement du flamenco. La plupart des objectifs du projet étant déjà remplis par d'autres institutions et organisations, jusque dans la propre ville de Jerez. Par exemple, le Centre andalou de flamenco, installé à Jerez, constitue la plus importante source de documentation mondiale sur cet art. Le centre de documentation prévu dans la ciudad del flamenco apparaît donc pour certains comme une aberrante redondance. Au niveau régional également, tant les conditions de production artistique que l'accueil touristique sont déjà bien développés. Aussi, face à cette absence de consultation, la Région créa l'Agence Andalouse pour le Développement du Flamenco et changa le nom de la Compagnie Andalouse de Danse pour « Ballet Flamenco de Andalucia », ce qui contribua à accentuer cette confrontation.

Les dernières nouvelles relatent toutefois le soutien du projet par l'actuelle majorité politique, même s'il devrait changer radicalement, tant dans sa forme que dans ses objectifs, 
avec une «meilleur coordination entre tous les acteurs et un nouveau nom rappelant la place de Jerez comme centre mondial du flamenco » (Pilar Sánchez Muñoz, in 20 Minutos, édition du 7 février 2008). Si fin 2007 la question du financement tournait encore en rond, la visite du président Jose Luis Rodriguez Zapaterro en août à Jerez initia une volonté gouvernementale de résoudre ce problème. Ainsi, c'est le directeur général de l'Institut National des Arts Scéniques et de la Musique, Juan Carlos Marset, qui affirmera que les travaux, arrêtés depuis plusieurs mois, sont « sur le point de recommencer et que leur fin est prévue pour 2011 » (20 Minutos, édition du 7 février 2008).

\section{Eléments de conclusion}

Le flamenco est une de ces formes musicales qui, au même titre que beaucoup d'autres (jazz, blues...), constitue bien plus qu'une dimension musicologique, artistique et même culturelle. Nous retrouvons en effet, de par son analyse, les indices et marqueurs qui nous apparaissent suffisants pour confirmer l'intérêt d'une attention particulière portée par les géographes. L'aménagement, comme nous l'avons montré, fait partie de ces domaines qui se nourrissent de la culture locale et de ses principes de fonctionnement. Aussi, penser et aménager la ville en Andalousie c'est compter avec la puissance flamenca à l'œuvre aujourd'hui. Elle nous apprend qu'une vision globale du territoire doit pouvoir se constituer autour des éléments concrets et pragmatiques, certes, mais également en intégrant les incertitudes et les difficultés d'acception des forces invisibles qui régissent la dynamique socioculturelle, économique et politique. Dans le concert de la planification andalouse, le flamenco est à la fois sur la scène, dans le public, en coulisse et absent de la manifestation. La question n'est donc plus de savoir s'il faut faire avec, mais comment agir en connaissance de cause.

Le couple musique/territoire, fruit d'interrelations complexes entre populations, pratiques culturelles et artistiques et degré d'ouverture sur l'extérieur, nous invite alors à penser que la musique à sa place aujourd'hui en géographie. Il existe un lien entre territoire et musique, cette dernière nécessitant de par son essence une double inscription : spatiale, en se référent à sa localisation géo-historique, et territoriale, en passant par les paradigmes socioculturels et identitaires qui la détermine. Debarbieux (2003) dira du territoire qu' « il participe toujours d'une vision du monde et d'une représentation auto-référencée et identitaire du groupe qui le construit ». Au sens où la musique est un produit identitaire, le territoire est donc producteur du sens musical puisqu'il régit lui-même la dynamique identitaire. En effet, le flamenco patrimonialisé parle du collectif qui le mobilise. Le territoire-acteur de cette construction patrimoniale fait ainsi du produit flamenco latent une ressource active. Alors clairement inscrite à la fois dans une concurrence internationale de l'offre culturelle et touristique et dans des conditions de construction complexes localisées, cette ressource devient territoriale au sens où ses perspectives théoriques semblent bien résister à son illustration empirique. La récente acceptation par l'UNESCO de la candidature de l'Espagne pour la déclaration du flamenco comme Patrimoine Culturel Immatériel de l'Humanité relance toutefois le débat. La lettre du 5 juin 2010 de la Communauté Autonome d'Andalousie, en partenariat avec les Régions d'Estrémadure et de Murcie, envoyée à l'ensemble des acteurs du flamenco pour leur demander d'adhérer au processus pose la question en ces termes : " toutes les adhésions sont importantes et contribuent à ce que le flamenco, grand ambassadeur de l'Espagne dans le monde, puissante ressource socioéconomique et pierre angulaire de notre identité culturelle, se convertisse officiellement 
en Patrimoine Immatériel de l'Humanité ». Si le processus de territorialisation de la ressource n'est pas unidirectionnel, mais se trouve plutôt dans un équilibre entre les potentialités du local et les exigences du global, cette proposition, qui ouvre la voie à de multiples interprétations, fait état de l'enjeu actuel qui gravite autour de notre problématique.

\section{Références bibliographiques}

Anderson B. ; Morton F. ; Revill G., 2005, "Editorial. Practice of music and sound", in Social \& Cultural Geography, vol.6, n5 (special), p.639-644.

Apprill C. et Dorier Apprill E., 1998, « Espaces et lieux du tango. La géographie d'une danse, entre mythe et réalité », in Le voyage inachevé...à Joël Bonnemaison, Paris, ORSTOM-Prodig, p.583-590.

Bandt R., Duffy M., MacKinnon D. (eds), 2007, Hearing Places: Sound Place Time Culture, Cambridge Scholars Publishing.

Bennett A.; Hawkins S., 2004, Music, space and place, Sheila Whiteley, Aldershot.

Berque A., 2000, "Acoustique n'est pas musique (et géométrie n'est pas géographie)", in L'Espace Géographique, $\mathrm{n}^{\circ}$, vol.2, 2000, p.279-280.

G. Bertrand, 1968, «Paysage et géographie physique globale. Esquisse méthodologique », Géographie Pyrénées et S.O., vol.39, n³, Toulouse, pp. 249-271.

Bonnemaison J., Copans J., 2000, La géographie culturelle, Cours de l'Université Paris IV Sorbonne, 1994-1997 (établi par Maud LASSEUR et Christel THIBAULT), Paris, éditions du Comité des travaux historiques et scientifiques (CHTS).

Byklum D., 1994, "Geography and Music, Making the Connection", in Journal of Geography, Geography Framework for the 1994 National Assessment of Educational Progress Geography for Life: National Geography Standards.

Caffarena A., 1964, Geografía del cante andaluz, Málaga.

Carney G.O., 1974, "Bluegrass Grows All Around: The Spatial Dimensions of a Country Music Style." Journal of Cultural Geography, vol.73, no. 4, 1974, p.34-55.

Carney G.O. (Dir.), 2003 [1978], The Sounds of People and Places: A Geography of American Music From Country to Classical and Blues to Bop, Lanham, MD: Rowman \& Littlefield, lére edition.

Connell J.; Gibson C., 2003, Sound Tracks: Popular Music, Identity and Place, Routledge.

Cook I. ; Crouch D. ; Naylor S. ; Ryan J. (Dir.), 2000, Cultural Turns/geographical Turns, Prentice Hall, Harlow.

Cruces Roldan C., 2002, Más allá de la música: antropología y flamenco, Sociabilidad, transmisión y patrimonio, Signatura Ediciones de Andalucía, Sevilla.

Deval F., 1989, Le flamenco et ses valeurs, Aubier.

Fanise P., 2002, « Vers une nouvelle Andalousie », Mediteria, n 16, P.24-25.

Ford L.R., 1971 "Geographic Factors in the Origin, Evolution, and Diffusion of Rock and Roll Music", in Journal of Geography, nº 70(8), p.455-464.

Foucher M., (Dir.), 1996, Les ouverture de l'opéra : une nouvelle géographie culturelle ?, Presses universitaires de Lyon.

Gironcourt (de) G., 1932, Une science nouvelle: la géographie musicale, Nancy.

Grimaldos A., 1998, Panorama del flamenco, FNAC-Espagne.

Grison L., 2000, "Espace et musique: répons de Boulez", in L'Espace Géographique, janvier 2000, p.87-89.

Guiguère H., 2005(a), "La patrimonialisacion como estrategia de apropiación política del flamenco en Jerez de la Frontera", in Gondas Pontasany M. ; Mendez Perez L. (Dir.), Politica Cultural: iniciativas de la administración, repuestas de los administrados, Asociación Andaluza de Antropología, Sevilla, 2005a, p.69-81.

Guiguère H., 2005(b)"El patrimonio inmaterial y sus apuestas: estudio comparativo de la politización y de la mercantilización de las culturas del flamenco y de las bodegas en Jerez de la Frontera", in Pereiro Pérez X. ; Sierra Rodríguez X.C. (Dir.), Patrimonio cultural: politizaciones y mercantilizaciones, Federación de Asociaciones de Antropología del Estado Español, Sevilla, 2005(b), p.101-113.

Guiu C. (Dir.), 2006, « Géographie et musiques : quelles perspectives ?», Géographie et cultures, n59.

Guiu C., 2009, «Les géographies sonores : rythmes et contrepoints », in Raibaud Y. (Dir.), Comment la musique vient au territoires?,MSHA, Pessac, p.29-59.

Gumuchian H. ; Pecqueur B., 2007, La ressource territoriale, Economica : Anthropos. 
Jacques Herzog et Pierre De Meuron, Projet architectural de la Ciudad del Flamenco à Jerez de la Frontera, 2004.

Kong L., 1996, "Popular music and a "sense of place" in Singapore", in Crossroads, n9, p.51-77.

Knight D.B., 2006, Landscape in music: space, place and time in the world great music, Lanhan, Rowman\&Littlefield Publisher.

Krim A., 1998, “"Get your kick on route 66!" A song map of post-war migration”, in Journal of cultural geography, $\mathrm{n}^{\circ} 18, \mathrm{p} .49-60$.

Krim A., 2007, Music and urban geography, Londres, Routledge.

Kruse R.J., 2003, "Imagine Strawberry Field as a place of pilgrimage", in Area, June, Vol.35, n², p.154-162.

Lacombe H., 2007, Géographie de l'opéra au XXè siècle, Fayard.

Ladmirault P., 1914, "Géographie musicale de l'Europe (2e Séance)", in La Revue Musicale S.I.M., 1er avril 1914, p.56.

Lamantia F., 2005, L'opéra dans l'espace français, Paris, Connaissance et savoir, 484p.

Lévy J., 1999, Le tournant géographique, Belin, Paris.

Lefranc P., 1998, Le cante jondo: le territoire, le problème des origines, les répertoires, Faculté des Lettres de Nice.

Leyshon A.; Matless D.; Revill G. (eds.), 1998, The place of music, Guilford Press, New York.

Macías M., 2007, Parole du sous-directeur du marketing du tourisme andalou, in La nueva Arbolea, N4, SeptDec.

Mairena A., 1976, Las confesiones de Antonio Mairena, ed. Sevilla.

Manfredi D., 1955, Geografia del cante jondo, Madrid.

Martín Martín M., 1995, "Los festivales flamencos", in Historia del flamenco, Tartesos, Sevilla, p.445-497.

Molina R., Espìn M., 1992, Flamenco de ida y vuelta, Ed. Guadalquivir, Sevilla.

Nash P.H., 1968, "Music region and regional music", Deccan geographer, vol.6 n², p.1-24.

Nash P.H. ; Carney G.O., 1996, "The seven themes of music geography", in The Canadian Geographer, n40(1), p.69-74.

Pailhé J., 1998, "Le jazz: mondialisation et territorialité", in Mappemonde, n51, vol.3, p.38-43.

Pailhé J., 2004, "La musique dans le processus identitaire en Europe centrale", in Annales de géographie, $\mathrm{n}^{\circ} 113$, p.445-468.

Raibaud Y., 2005a, Territoires musicaux en régions. L'émergence des musiques amplifiées en Aquitaine, MSHA, Pessac.

Raibaud Y. (Dir.), 2009, Comment la musique vient au territoires ?,MSHA, Pessac.

Romagnan J. M., 2000, «La musique : un nouveau terrain pour les géographes », in Géographie et culture $\mathrm{n}^{\circ} 36$, pp.107-126.

Sandoval B. et G., 1998, Le flamenco, entre révolte et passion, Milan.

Smith S.J., 2000a, "Performing (Sound)world", Environment and Planning D: Society and Space, n¹8, p.61537.

Steingress G., 2002, « El flamenco commo patrimonio cultural o una construction artificial mas de la identidad andaluza », Anduli, n¹, p.43-65.

Suarez Japon J.M., 2002, Geografia del flamenco, Universidad Internacional de Andalucia.

Vauchey J.-P., 1987, « Pour une géographie des espaces sonores », Netcom, vol. 1, n², p. 235-246.

Vega Lopez J. (de), 2007, "De las patrimonializaciones del flamenco", in La Nueva Arbolea, N4, sept-dec, p.46-49.

Waterman S.; Brunn S.D. (eds.), 2006, “Geography and music”, numéro spécial de la revue GeoJournal, nº6, Springer.

\section{Glossaire}

Cantaor : Interprète de chant flamenco (en espagnol, chanteur se dit cantador ou cantante).

Cante jondo : littéralement "chant profond".

Ciudad del flamenco : littéralement « ville du flamenco"

Duende : feeling, magie du l'instant flamenco.

Jaleo : soutient de la peña aux artistes via le palmeo (jeu des mains), le « olé » et autres encouragements.

Palo : style, variante, genre, à l'intérieur du répertoire flamenco. 
Payo : non-gitan.

Peña : membres de la famille et amis formant un groupe cohérent d'accompagnement. Estilos : "petits sons", "touches". 